\title{
Bioinformatics analysis of microRNA expression between patients with and without latent tuberculosis infections
}

\author{
YANG LU ${ }^{1 *}$, XINMIN WANG ${ }^{2 *}$, HONGCHANG DONG $^{3 *}$, XIAOFANG WANG $^{1}$, PU YANG $^{1}$, \\ LING HAN $^{1}$, YINGZI WANG ${ }^{1}$, ZHIHONG ZHENG ${ }^{1}$, WANJIANG ZHANG ${ }^{1}$ and LE ZHANG $^{1}$ \\ ${ }^{1}$ Department of Pathophysiology, The Key Laboratory for Xinjiang Endemic and Ethnic Diseases; \\ ${ }^{2}$ Department of Urinary Surgery, The First Affiliated Hospital, Medical College of Shihezi University; \\ ${ }^{3}$ Department of Biochemistry, The Key Laboratory for Xinjiang Endemic and Ethnic Diseases, \\ Medical College of Shihezi University, Shihezi, Xinjiang 832000, P.R. China
}

Received September 12, 2018; Accepted March 6, 2019

DOI: $10.3892 /$ etm.2019.7424

\begin{abstract}
Tuberculosis (TB) is a globally prevalent infectious disease. The mechanisms of latent TB infection (LTBI) remain to be fully elucidated and may provide novel approaches for diagnosis. As therapeutic targets and molecular diagnostic markers, microRNAs (miRs) have been studied and utilized in various diseases. In the present study, the differentially expressed miRs (DEMs) in LTBI were screened and analyzed to determine the underlying mechanisms and identify potential biomarkers, thereby contributing to the diagnosis of LTBI. The GSE25435 and GSE29190 datasets from Gene Expression Omnibus were selected for analysis. The 2 datasets were analyzed individually using the Bioconductor package to screen the DEMs with specific cut-off criteria $[\mathrm{P}<0.01$ and $\mid \log$ (fold change) $\mid \geq 1$ ]. Target gene prediction and interaction network construction were performed using Targetscan, the Search Tool for the Retrieval of Interacting Genes and Proteins and Cytoscape individually, and were merged using the latter tool. The hub genes were finally selected based on their degree of connectivity (DC). Gene Ontology and Kyoto Encyclopedia of Genes and Genomes (KEGG) pathway analyses were performed using the KEGG and GENCLIP. A total of 144 DEMs were identified from the 2 datasets. By exploring the overlapping miRs in the two datasets, Homo sapiens (hsa)-miR-29a and hsa-miR-15b were identified to be decreased, while hsa-miR-576-5p, hsa-miR-500 and hsa-miR-155 were identified to be upregulated. hsa-miR-500a-3p and hsa-miR-29a-3p,
\end{abstract}

Correspondence to: Professor Le Zhang, Department of Pathophysiology, The Key Laboratory for Xinjiang Endemic and Ethnic Diseases, Medical College of Shihezi University, 221 Beisi Road, Shihezi, Xinjiang 832000, P.R. China

E-mail: 1257067540@qq.com

*Contributed equally

Key words: latent tuberculosis infection, bioinformatics analysis, microRNA, diagnosis, therapeutic target as well as 4 genes, namely cell division cycle (CDC) 42, actin $\alpha 1$, skeletal muscle (ACTA1), phosphatase and tensin homolog (PTEN) and fos proto-oncogene (FOS), were selected as the key factors in this regulatory network. A total of 9 signaling pathways, including phosphoinositide-3 kinase (PI3K)/AKT and 11 biological processes, were identified to be associated with LTBI. In conclusion, the present analysis identified hsa-miR-500a-3p and hsa-miR-29a-3p, as well as CDC42, ACTA1, PTEN and FOS, as the most promising biomarkers and therapeutic candidates for LTBI. The PI3K/AKT signaling pathway is the key signaling pathway implicated in LTBI, and an in-depth investigation of the efficiency of PI3K/AKT signaling inhibitors may be used to prevent a chronic state of infection in LTBI.

\section{Introduction}

Tuberculosis (TB) is a global infectious disease caused by infection with the pathogen Mycobacterium tuberculosis (MTB) and poses a serious risk to human health. It is a contagious disease that spreads by inhalation of bacteria contained in the breath of an infected individual. China ranks second out of the countries with a high TB burden. According to the 2017 World Health Organization (WHO) global TB report (1), TB is the ninth most lethal disease in the world and ranks first among infectious diseases, with a mortality rate much higher than that of acquired immune deficiency syndrome. Latent TB infection (LTBI) is defined as a state of persistent immune response to stimulation by MTB antigens with no evidence of any clinical manifestations of active TB (2). Within the population of individuals with LTBI, most cases remain asymptomatic and are not contagious; however, $5-10 \%$ of those who are infected progress to active TB disease and become contagious $(2,3)$. LTBI is a continuum between self-healed and asymptomatic (4). At present, there are no unified diagnostic criteria for LTBI. The current method used is the tuberculin skin test (TST) and interferon- $\gamma$ release assay (IGRA), however both have certain limitations, which include poor specificity and high cost of diagnosis, as well as being complex techniques (5-8). At present, the WHO recommends the use of the IGRA to detect LTBI in middle- and high-income countries with greater resources (9), while the TST 
is recommended in countries with limited resources. Therefore, it is necessary to develop a rapid, low-cost, non-invasive and efficient diagnostic method for the prevention and control of TB.

MicroRNAs (miRs) are a class of non-coding RNAs composed of 21-23 nucleotides, which are not translated into proteins. They are cut from precursor miRs with 6,070 nucleotides and a hairpin structure. The major function of miRs is to regulate gene expression at the post-transcriptional level, primarily by binding to the 3'-untranslated region of the mRNAs of their target genes to degrade the mRNA or inhibit its translation, thus inhibiting the expression of the target genes (10). When disease occurs, the specific miRs of the lesion organs may be released into the blood. In numerous diseases, the miR content in the blood is significantly different from that of healthy individuals (11). To date, miRs as molecular diagnostic markers have been studied and applied in cancer, diabetes, psychiatric disorders, heart disease and various infectious diseases (12-16). More recent evidence suggests the use of miRs as biomarkers for LTBI. However, compared with the well-known role of certain miRs in cancer, the biological functions and diagnostic utility of miRs in LTBI remain largely elusive. Wu et al (17) identified that the expression of Homo sapiens (hsa)-miR-142-3p and hsa-miR-21-5p was enhanced in the peripheral blood samples from patients with LTBI compared with patients with TB. Meng et al (18) indicated that miR-93-3p is a potential diagnostic marker for distinguishing LTBI from active TB. Using bioinformatics to further analyze gene expression data, the present study unveiled certain characteristics and mechanisms involved in TB latency, which may provide a theoretical basis for the early diagnosis and treatment of, and experimental research into TB (19), and may serve as a reference for further investigation of molecular events indicative of LTBI.

In previous decades, microarray analysis has been frequently used to identify candidate biomarkers and therapeutic targets by studying changes in non-coding RNA and gene expression profiles at a genome-wide scale (20). A previous study on diagnosing latent $\mathrm{TB}$ infection identified dysregulated miRs and associated pathways of LTBI (21). However, only a small number of miRs have been identified to be significantly differentially expressed between patients with and without LTBI. Due to these discordant results, the reliability of these data for further development into useful clinical diagnostic biomarkers and therapeutic targets for TB is limited. It has been well recognized that small sample sizes, different microarray platforms and different statistical methods are among the limiting factors contributing to the discordant results. To overcome this limitation, meta-analysis represents a powerful approach to combine datasets from different studies to improve the reliability and generalizability of the results by increasing the statistical power. Meta-analysis of gene expression data or non-coding RNA profiles has yielded novel biological insight, and has identified more robust and reliable candidate biomarkers and therapeutic targets.

In the present study, to increase the understanding of latent TB infection, the GSE25435 and GSE29190 datasets were downloaded from the GEO database and analyzed using $\mathrm{R}$ tools for differentially expressed miRs (DEMs). The regulatory network of these DEMs and their target genes was constructed to explore the potential regulatory interactions involved in LTBI. Enrichment analysis of these target genes in Gene Ontology (GO) terms in the category biological process (BP), molecular function (MF), cellular component (CC) and Kyoto Encyclopedia of Genes and Genomes (KEGG) pathways was performed for further identification of LTBI-associated pathways and molecular mechanisms.

\section{Materials and methods}

Selection of microarray datasets. To retrieve suitable LTBI-associated miR profiling data for the meta-analysis, a web-based search in the Gene Expression Omnibus database (GEO; http://www.ncbi.nlm.nih.gov/geo) and ArrayExpress (http://www.ebi.ac.uk/arrayexpress) database was performed using the key words 'latent TB infection' and 'microRNA'. In total, 5 array datasets were identified in the GEO and ArrayExpress databases. The datasets were manually reviewed and only those fulfilling the following criteria were included for further analysis: i) miR expression profiling by array; and ii) paired samples from patients with and without LTBI. Finally, a total of 2 datasets, GSE25435 and GSE29190, were selected for analysis. miR expression data of peripheral blood mononuclear cells (PBMCs) from 6 donors with LTBI and 3 healthy controls are included in GSE29190. GSE25435 contains miR expression data from PBMCs of 3 donors with LTBI and 3 healthy controls.

Screening of DEMs. The 2 datasets were analyzed individually by using the Bioconductor package to screen the DEMs with specific cut-off criteria $[\mathrm{P}<0.01$ and $\mid \log$ fold change $(F C) \mid \geq 1]$ (22). Paired samples t-tests and the FC method were used to obtain DEMs. The DEMs of the 2 datasets were then uploaded to the online VENN tool (http://bioinformatics.psb. ugent.be/webtools/Venn/) to identify the overlapping miRs (23).

Target gene prediction and network construction. The prediction of target genes of different miRs was performed by using TargetScan (http://www.targetscan.org/vert_71) (24). A cumulative weighted context++ score online $>-0.5$ was set as the standard for screening target genes, as previously described (24). The target genes were then uploaded to the online database Search Tool for the Retrieval of Interacting Genes and Proteins (STRING; string-db.org) (25) to obtain the interaction information for these target genes. The miR targeting data and target gene interaction data were imported to Cytoscape, which was used to visualize and merge networks. The key factors were finally selected based on their DC $(26,27)$.

GO and KEGG pathway analysis. Pathway enrichment analysis was performed using KEGG, a collection of databases on genomes, biological pathways, diseases, drugs and chemical substances (28). Target genes identified in the abovementioned analysis were uploaded to GENCLIP (ci.smu.edu. cn/GenCLiP2/analysis.php) to perform the GO enrichment analysis in the categories $\mathrm{BP}, \mathrm{CC}$ and $\mathrm{MF}$, and $\mathrm{P}<0.01$ was set as the cut-off criterion (29).

\section{Results}

Individual microarray data analysis of DEMs. To identify DEMs in patients with LTBI vs. healthy individuals, $\mathrm{P}<0.01$ 

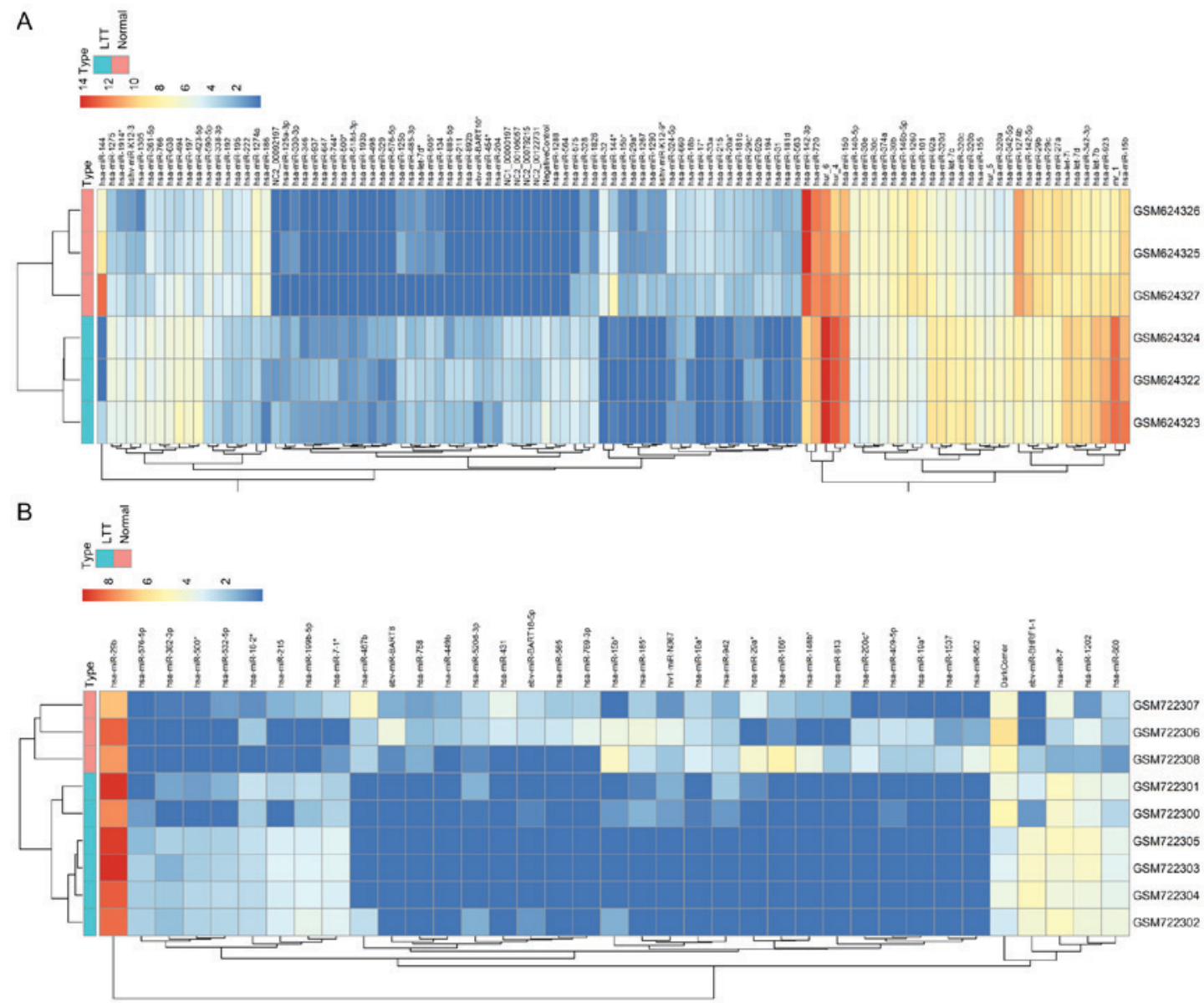

Figure 1. Hierarchical clustering of the DEMs from patients with and without LTBI. The color scale at the top illustrates the relative expression level of an mRNA. Red represents a high relative expression and blue represents a low relative expression. (A) DEMs of GSE25435. (B) DEMs of GSE29190. DEM, differentially expressed miR; LTB, latent tuberculosis; miR, microRNA; hsa, Homo sapiens.

and $\mid \log \mathrm{FCl} \geq 1$ were set as cut-off criteria when screening the miR array profiles. A total of 107 miRs, including 44 downregulated and 63 upregulated miRs, were identified to be dysregulated in the GSE25435 dataset (Table I). The GSE29190 dataset was also analyzed, revealing only 37 DEMs, including 24 downregulated and 13 upregulated miRs (Table II). The clustering heatmap analysis of these two datasets indicated a highly significant difference between LTBI and normal patients, meriting further analysis (Fig. 1).

Meta-analysis of the dysregulated miRs provides 5 hub $m i R s$. Due to the limitations of the analyses of the individual datasets, the overlapping miRs between the two datasets were selected for the analysis of hub miRs. By Venn diagram analysis, hsa-miR-29a and hsa-miR-15b were identified as being downregulated in the two datasets (Fig. 2A), while hsa-miR-576-5p, hsa-miR-500 and hsa-miR-155 were identified as being upregulated (Fig. 2B).

Construction of the molecular network provides complex regulatory interactions. The target genes of the abovementioned miRs were assessed using the online tool Targetscan with a pre-defined standard. A total of 216 potential target genes were identified in the present analysis and they are listed in Table III. To illustrated the direct regulatory associations between the hub miRs and their target genes, as well as interactions of these target genes, the network of these factors was constructed using Cytoscape. A complex regulatory network was obtained, suggesting that dysregulation of miRs during LTBI may cause a widespread dysregulation in gene expression. In addition, dysregulation of miRs during LTBI may lead to functional disorders via signaling pathways or BPs. By exploring the regulatory network, the key miRs and genes may be selected based on the DC in the network. A total of 2 miRs, namely hsa-miR-500a-3p (degree, 54) and hsa-miR-29a-3p (degree, 77), as well as 4 genes, namely cell division cycle (CDC)42 (degree, 21), actin $\alpha 1$, skeletal muscle (ACTA1; degree, 21), phosphatase and tensin homolog (PTEN; degree, 21) and FOS (degree, 21), were selected as the core factors that may have principal roles in LTBI (Fig. 3).

Functional and pathway analysis. To investigate the signaling pathways that are potential associated with LTBI, the target genes were subjected to KEGG pathway enrichment analysis. A total of 9 pathways were enriched by the target genes (Fig. 4), including 'protein digestion and absorption', 'extracellular matrix-receptor interaction' and 'phosphoinositide-3 kinase (PI3K)/AKT signaling pathway'. In addition, inclusion of small cell lung cancer pathways in the present analysis indicated that LTBI may be associated with the development of small cell lung cancer. The 185 target genes were subsequently subjected to GO term enrichment analysis which revealed that 90 target genes were enriched in the following pathways: 
Table I. Differentially expressed miRs in the GSE25435 dataset.

\begin{tabular}{|c|c|c|c|c|}
\hline $\operatorname{miR}$ & $\log \mathrm{FC}$ & AveExpr & $\mathrm{t}$ & P-value \\
\hline hsa-miR-32 & -4.83703 & 2.490441 & -32.8327 & $2.86 \times 10^{-6}$ \\
\hline hsa-let-7d & 1.605658 & 8.870986 & 25.95471 & $7.72 \times 10^{-6}$ \\
\hline hsa-miR-1260 & -3.67093 & 6.940923 & -21.9504 & $1.56 \times 10^{-5}$ \\
\hline hsa-miR-181d & -2.24959 & 1.176625 & -21.3805 & $1.75 \times 10^{-5}$ \\
\hline NC1_00000197 & 3.79419 & 1.948923 & 21.37752 & $1.75 \times 10^{-5}$ \\
\hline hsa-miR-1288 & 4.12176 & 2.112708 & 20.71065 & $2.00 \times 10^{-5}$ \\
\hline hur_6 & 2.153942 & 13.10695 & 19.77886 & $2.43 \times 10^{-5}$ \\
\hline NC2_00079215 & 3.222861 & 1.663258 & 15.16582 & $7.39 \times 10^{-5}$ \\
\hline hsa-miR-17* & -3.75792 & 1.930786 & -14.9806 & $7.78 \times 10^{-5}$ \\
\hline NC2_00106057 & 3.685307 & 1.894481 & 14.56989 & $8.74 \times 10^{-5}$ \\
\hline hsa-miR-142-5p & -3.15876 & 8.353603 & -14.0788 & 0.000101 \\
\hline hsa-let-7i & 1.002891 & 9.400425 & 13.92367 & 0.000106 \\
\hline hsa-miR-30e & -2.12857 & 6.050006 & -13.8563 & 0.000108 \\
\hline hsa-miR-30b & -1.24457 & 7.124084 & -13.295 & 0.000128 \\
\hline hsa-miR-454* & 2.311672 & 1.207664 & 12.96173 & 0.000142 \\
\hline hsa-miR-1274b & -3.22595 & 9.539236 & -12.8303 & 0.000148 \\
\hline hsa-miR-20a* & -3.09852 & 1.688503 & -12.5566 & 0.000162 \\
\hline hsa-miR-564 & 3.682902 & 1.893279 & 11.84041 & 0.000207 \\
\hline hsa-miR-30c & -1.47533 & 6.141081 & -11.4563 & 0.000237 \\
\hline hsa-miR-518d-3p & 1.098449 & 0.601052 & 11.36622 & 0.000245 \\
\hline hsa-miR-142-3p & -4.08985 & 11.79739 & -11.1118 & 0.000269 \\
\hline hsa-miR-215 & -2.79467 & 2.085103 & -10.6335 & 0.000323 \\
\hline hsa-miR-320c & 2.710049 & 6.808952 & 10.40018 & 0.000353 \\
\hline hsa-miR-29b & -2.6684 & 7.916884 & -10.2334 & 0.000378 \\
\hline hsa-miR-193b & 1.481897 & 0.792776 & 10.23095 & 0.000378 \\
\hline hsa-miR-505* & 2.825782 & 2.002488 & 10.08595 & 0.000401 \\
\hline hsa-miR-1274a & -3.96165 & 5.117785 & -10.0041 & 0.000415 \\
\hline hsa-miR-320d & 2.530531 & 7.386848 & 9.966743 & 0.000421 \\
\hline hsa-miR-194 & -2.9415 & 1.573092 & -9.87762 & 0.000437 \\
\hline mr_1 & 4.009616 & 10.57346 & 9.598379 & 0.000491 \\
\hline hsa-miR-197 & 1.865861 & 5.328528 & 9.377518 & 0.00054 \\
\hline hsa-miR-33a & -3.71272 & 1.90819 & -8.53538 & 0.000792 \\
\hline hsa-miR-374a & -1.05367 & 5.99272 & -8.48323 & 0.000812 \\
\hline hsa-miR-320b & 2.595755 & 6.879281 & 8.346227 & 0.000867 \\
\hline hsa-miR-211 & 2.928911 & 1.516283 & 8.276387 & 0.000897 \\
\hline NC2_00122731 & 3.230483 & 1.667069 & 8.074902 & 0.000990 \\
\hline hsa-miR-29c & -1.60822 & 8.001147 & -7.87047 & 0.001098 \\
\hline hsa-miR-195 & -1.58473 & 3.691033 & -7.80122 & 0.001138 \\
\hline hsa-let-7b & 2.253171 & 9.216569 & 7.57616 & 0.001279 \\
\hline hsa-miR-563 & -1.93468 & 1.56909 & -7.14915 & 0.001612 \\
\hline hsa-miR-140-5p & -1.35331 & 5.635543 & -7.11292 & 0.001645 \\
\hline hsa-miR-423-5p & 2.546925 & 5.162408 & 7.075481 & 0.001679 \\
\hline hsa-miR-892b & 2.908221 & 1.505938 & 6.779481 & 0.001988 \\
\hline hsa-let-7c & 1.591322 & 7.493733 & 6.714825 & 0.002065 \\
\hline hsa-miR-181c & -2.61189 & 1.740467 & -6.71423 & 0.002066 \\
\hline hsa-miR-31 & -2.69031 & 1.538948 & -6.55738 & 0.002267 \\
\hline hur_4 & 2.724996 & 11.79659 & 6.333833 & 0.002596 \\
\hline hsa-miR-155 & 1.135612 & 6.034357 & 6.210813 & 0.002802 \\
\hline hsa-miR-720 & -1.17258 & 11.34608 & -5.89947 & 0.003420 \\
\hline hsa-miR-222 & -1.33272 & 3.65274 & -5.83189 & 0.003575 \\
\hline hsa-miR-186 & -4.09024 & 3.67533 & -5.76641 & 0.003734 \\
\hline hsa-miR-101 & -2.5019 & 6.584804 & -5.69109 & 0.003927 \\
\hline
\end{tabular}


Table I. Continued.

\begin{tabular}{|c|c|c|c|c|}
\hline $\operatorname{miR}$ & $\log \mathrm{FC}$ & AveExpr & $\mathrm{t}$ & P-value \\
\hline hsa-miR-204 & 2.505782 & 1.304719 & 5.150187 & 0.005734 \\
\hline hsa-miR-134 & 2.026807 & 1.755041 & 4.931725 & 0.006741 \\
\hline hsa-miR-1290 & -1.70206 & 0.902855 & -4.8857 & 0.006979 \\
\hline hsa-miR-575 & 3.799179 & 2.716106 & 4.762392 & 0.007670 \\
\hline hsa-miR-937 & 1.689168 & 0.896412 & 4.735594 & 0.007830 \\
\hline hsa-miR-320a & 1.789151 & 6.023945 & 4.670974 & 0.008235 \\
\hline hsa-miR-590-5p & -1.58344 & 4.687364 & -4.6666 & 0.008263 \\
\hline hsa-miR-1275 & 2.152322 & 4.508394 & 4.391423 & 0.010302 \\
\hline NC2_00092197 & 2.244662 & 1.174159 & 4.37517 & 0.010440 \\
\hline hsa-miR-500* & 1.085714 & 0.806853 & 4.324164 & 0.010888 \\
\hline hur_5 & 1.581846 & 5.898678 & 4.300761 & 0.011102 \\
\hline hsa-miR-766 & 1.2936 & 4.933253 & 4.282655 & 0.011270 \\
\hline hsa-miR-1305 & 3.51088 & 3.923818 & 4.184017 & 0.012244 \\
\hline hsa-miR-485-3p & 2.264246 & 1.974846 & 4.137144 & 0.012742 \\
\hline hsa-miR-29c* & -1.41527 & 2.134653 & -4.08565 & 0.013317 \\
\hline hsa-miR-328 & 1.533516 & 3.063469 & 4.035669 & 0.013904 \\
\hline hsa-miR-1826 & 2.542979 & 2.772236 & 4.009905 & 0.014219 \\
\hline hsa-miR-923 & 2.864446 & 9.516405 & 3.979143 & 0.014606 \\
\hline hsa-miR-92a & 1.491981 & 8.018584 & 3.940985 & 0.015104 \\
\hline hsa-miR-1267 & -2.09638 & 1.132907 & -3.91896 & 0.015401 \\
\hline hsa-miR-885-5p & 2.463596 & 1.283626 & 3.881055 & 0.015927 \\
\hline hsa-miR-144 & -7.93309 & 5.22873 & -3.85587 & 0.016289 \\
\hline hsa-miR-660 & -1.32829 & 2.555425 & -3.75816 & 0.017787 \\
\hline hsa-let-7d* & 1.904947 & 1.863162 & 3.758015 & 0.017790 \\
\hline hsa-miR-647 & 1.663436 & 0.883546 & 3.748012 & 0.017952 \\
\hline hsa-miR-342-3p & 1.119628 & 8.920874 & 3.645006 & 0.019731 \\
\hline hsa-miR-15b* & -1.18389 & 0.868758 & -3.5706 & 0.021146 \\
\hline hsa-miR-144* & -4.64489 & 2.712225 & -3.53154 & 0.021937 \\
\hline kshv-miR-K12-9* & -1.76769 & 1.026389 & -3.48719 & 0.022877 \\
\hline hsa-miR-15b & 1.65062 & 10.49454 & 3.437951 & 0.023976 \\
\hline hsa-miR-638 & 1.600023 & 4.980986 & 3.434029 & 0.024066 \\
\hline hsa-miR-744* & 1.677503 & 0.890579 & 3.371348 & 0.025564 \\
\hline hsa-miR-346 & 1.392836 & 0.748246 & 3.330718 & 0.026593 \\
\hline hsa-miR-324-5p & -2.61219 & 2.446374 & -3.28872 & 0.027707 \\
\hline hsa-miR-498 & 1.277597 & 0.690626 & 3.285391 & 0.027798 \\
\hline hsa-miR-329 & 1.335942 & 0.719799 & 3.261694 & 0.028454 \\
\hline hsa-miR-125a-3p & 1.424537 & 1.12041 & 3.210039 & 0.029946 \\
\hline hsa-miR-192 & -1.44406 & 3.520292 & -3.20944 & 0.029964 \\
\hline hsa-miR-18b & -1.96872 & 2.472096 & -3.1976 & 0.030319 \\
\hline hsa-miR-27a & -1.44475 & 8.246285 & -3.19705 & 0.030335 \\
\hline hsa-miR-92b & -1.58151 & 2.150902 & -3.18198 & 0.030795 \\
\hline hsa-miR-150 & 1.084065 & 11.60013 & 3.12891 & 0.032478 \\
\hline hsa-miR-342-5p & 1.584302 & 6.234256 & 3.120644 & 0.032750 \\
\hline hsa-miR-29a* & -1.22069 & 0.662175 & -3.08183 & 0.034061 \\
\hline hsa-miR-576-5p & 1.233511 & 0.668583 & 3.056434 & 0.034952 \\
\hline ebv-miR-BART10* & 1.833523 & 0.968589 & 3.013552 & 0.036519 \\
\hline hsa-miR-1914* & 2.359262 & 4.149506 & 2.986318 & 0.037556 \\
\hline hsa-miR-330-3p & 1.331046 & 1.394026 & 2.970476 & 0.038175 \\
\hline hsa-miR-494 & 2.245492 & 5.43426 & 2.961052 & 0.038548 \\
\hline hsa-miR-361-5p & 1.350614 & 4.881747 & 2.92993 & 0.039813 \\
\hline hsa-miR-125b & 2.025984 & 2.170862 & 2.884837 & 0.041731 \\
\hline hsa-miR-338-3p & -1.63845 & 4.620754 & -2.78552 & 0.046342 \\
\hline
\end{tabular}


Table I. Continued.

\begin{tabular}{lllll}
\hline $\operatorname{miR}$ & $\operatorname{logFC}$ & AveExpr & $\mathrm{t}$ & P-value \\
\hline hsa-miR-146b-5p & -1.29146 & 6.920841 & -2.75528 & 0.047860 \\
\hline
\end{tabular}

$\log \mathrm{FC}, \log$ fold change; AveExpr, average expression; miR, microRNA; hsa, Homo sapiens, *complementary strand of the microRNA.

Table II. Differentially expressed miRs in the dataset GSE29190.

\begin{tabular}{|c|c|c|c|c|}
\hline $\operatorname{miR}$ & $\log \mathrm{FC}$ & AveExpr & $\mathrm{t}$ & P-value \\
\hline hsa-miR-18a* & -2.51535 & 0.9441 & -15.4559 & $6.85 \times 10^{-7}$ \\
\hline hsa-miR-758 & -1.24903 & 0.487935 & -10.0342 & $1.46 \times 10^{-5}$ \\
\hline hsa-miR-185* & -2.498 & 1.185303 & -5.30749 & 0.000938 \\
\hline hsa-miR-199b-5p & 2.216376 & 2.08776 & 4.343231 & 0.002984 \\
\hline hsa-miR-16-2* & 1.558178 & 1.935857 & 4.180525 & 0.003676 \\
\hline hsa-miR-1202 & 2.150841 & 3.293889 & 4.041271 & 0.004408 \\
\hline hsa-miR-7 & 1.917814 & 3.758687 & 3.950336 & 0.004971 \\
\hline hsa-miR-660 & 1.536002 & 2.837873 & 3.529016 & 0.008814 \\
\hline hsa-miR-7-1* & 1.79193 & 2.285376 & 3.46859 & 0.009588 \\
\hline hsa-miR-487b & -2.48633 & 1.308742 & -3.28236 & 0.012470 \\
\hline hsa-miR-409-5p & -1.5458 & 0.669419 & -3.22662 & 0.013503 \\
\hline hsa-miR-200c* & -1.80803 & 0.674268 & -3.12735 & 0.015573 \\
\hline hsa-miR-613 & -1.11381 & 0.442862 & -3.11342 & 0.015889 \\
\hline hsa-miR-431 & -2.10028 & 0.771685 & -3.09273 & 0.016372 \\
\hline hsa-miR-19a* & -1.48092 & 0.565233 & -3.06806 & 0.016968 \\
\hline hsa-miR-186* & -2.5773 & 0.930693 & -3.04035 & 0.017664 \\
\hline ebv-miR-BART8 & -1.86635 & 0.693708 & -2.98042 & 0.019276 \\
\hline hsa-miR-585 & -1.52776 & 0.594644 & -2.97825 & 0.019337 \\
\hline ebv-miR-BHRF1-1 & 2.864032 & 2.647555 & 2.977097 & 0.019370 \\
\hline hsa-miR-576-5p & 1.428135 & 1.023683 & 2.942374 & 0.020379 \\
\hline hsa-miR-942 & -1.45888 & 1.013753 & -2.92631 & 0.020864 \\
\hline hsa-miR-449b & -1.03809 & 0.422371 & -2.90996 & 0.021370 \\
\hline hsa-miR-29a* & -2.36068 & 0.96128 & -2.89317 & 0.021904 \\
\hline hsa-miR-769-3p & -1.81466 & 0.676479 & -2.87074 & 0.022639 \\
\hline hsa-miR-362-3p & 1.258509 & 0.984151 & 2.859353 & 0.023021 \\
\hline hsa-miR-500* & 1.483617 & 1.126051 & 2.787552 & 0.025596 \\
\hline hiv1-miR-N367 & -1.63534 & 0.967286 & -2.70054 & 0.029123 \\
\hline ebv-miR-BART18-5p & -1.43074 & 0.748889 & -2.57279 & 0.035240 \\
\hline hsa-miR-148b* & -1.72537 & 0.646717 & -2.55501 & 0.036190 \\
\hline hsa-miR-532-5p & 1.417317 & 1.319251 & 2.552325 & 0.036336 \\
\hline hsa-miR-15b* & -2.3785 & 1.211755 & -2.54192 & 0.036907 \\
\hline hsa-miR-1537 & -1.0943 & 0.436358 & -2.5317 & 0.037477 \\
\hline hsa-miR-215 & 1.887456 & 1.812279 & 2.514436 & 0.038460 \\
\hline hsa-miR-662 & -1.57886 & 0.59788 & -2.4756 & 0.040769 \\
\hline hsa-miR-29b & 1.103322 & 8.302687 & 2.406804 & 0.045214 \\
\hline hsa-miR-520d-3p & -1.23035 & 0.943423 & -2.34466 & 0.049651 \\
\hline
\end{tabular}

$\log \mathrm{FC}, \log$ fold change; AveExpr, average expression; miR, microRNA; hsa, Homo sapiens; ebv, Epstein-Barr.

'Structural molecule activity', 'cardiovascular system development', 'organ morphogenesis', 'negative regulation of nitrogen compound metabolic process' with $\geq 15$ target genes enriched in several of these functional pathways, the most relevant of which were the 'cardiovascular system development' and 'circulatory system development' (Fig. 5). 

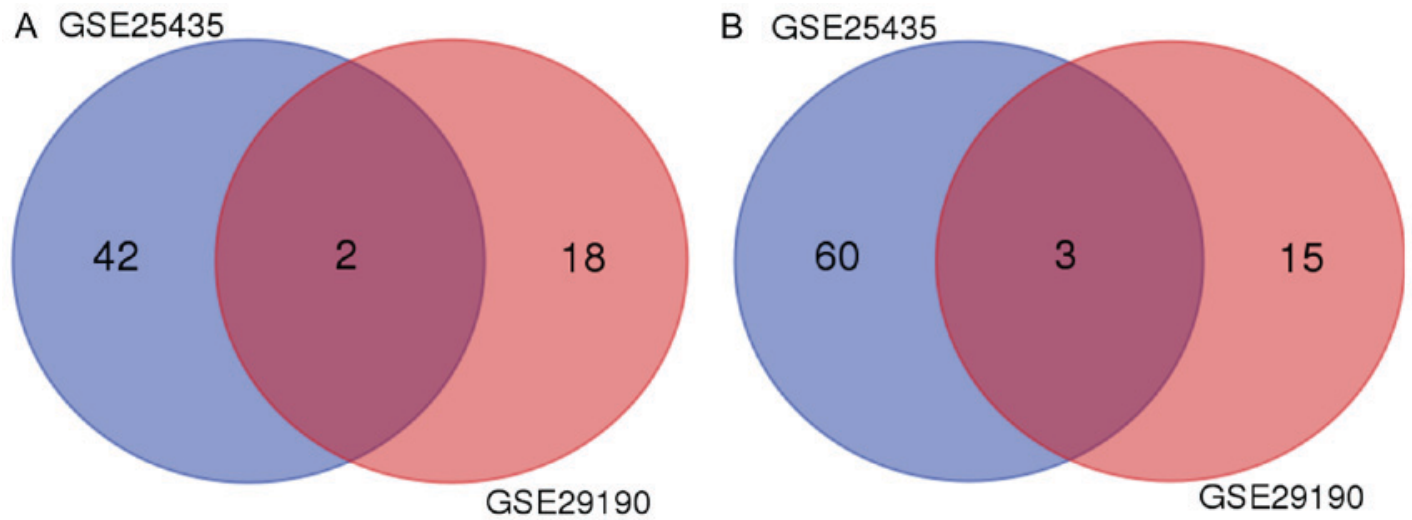

Figure 2. Venn diagram of (A) downregulated microRNAs and (B) upregulated microRNAs from the 2 datasets.

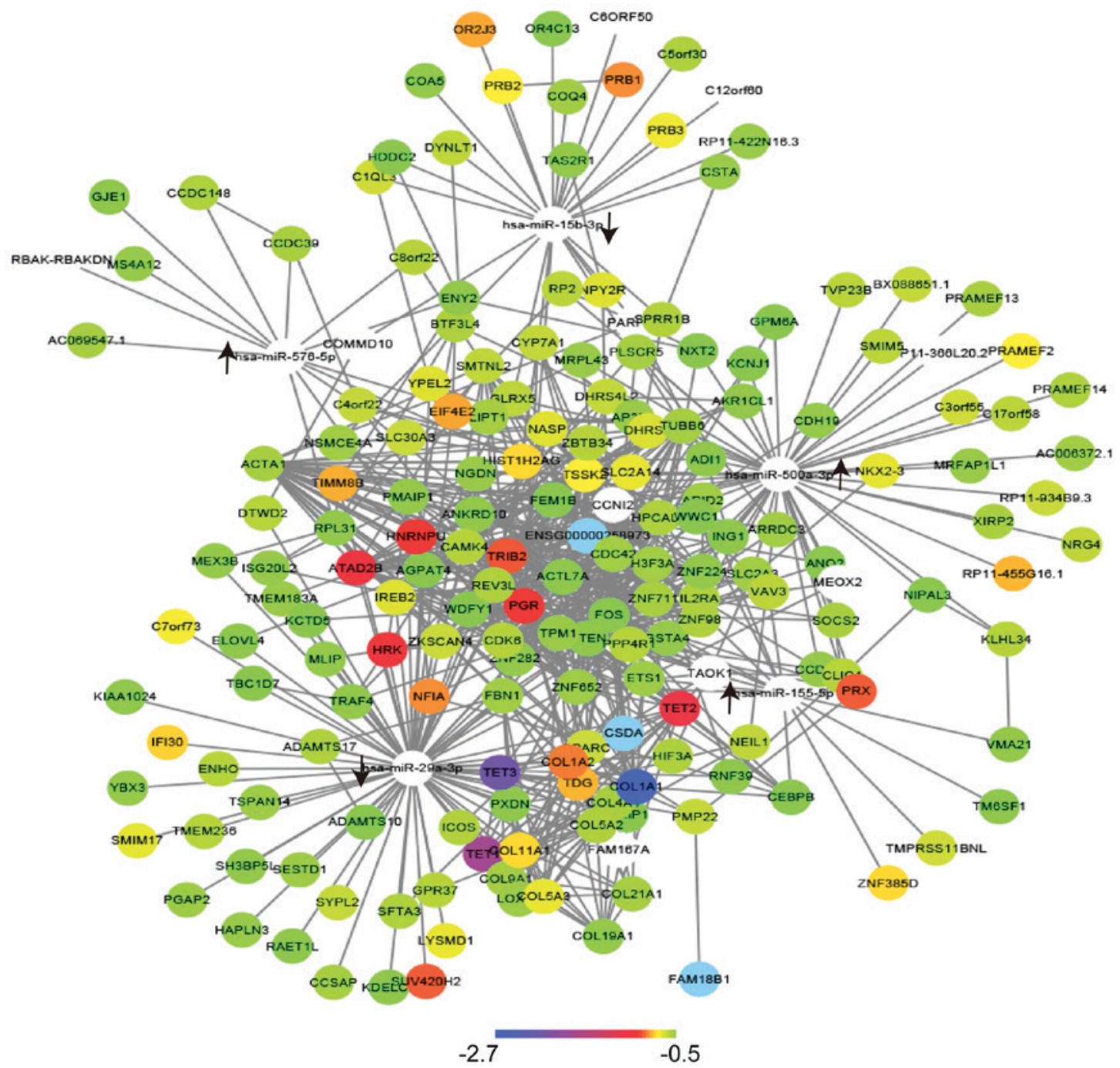

Cumulative weighted context++ score

Figure 3. Regulatory and interaction networks of the differentially expressed miRs and their target genes. The upward arrow indicates the upregulation of the miRs and the downward arrow indicates the downregulation of the miRs. The color scale presented at the bottom illustrates the cumulative weighted context++ scores of the target genes. miR, microRNA; hsa, Homo sapiens.

\section{Discussion}

The incomplete understanding of the underlying mechanisms and deficiencies in the diagnosis and treatment make it difficult to prevent and cure LTBI. Various host factors are included in this complex process. In the present study, miRs, a type of non-coding RNAs, were the focus, as they have been applied in multiple fields of life science and medicine. Microarray 


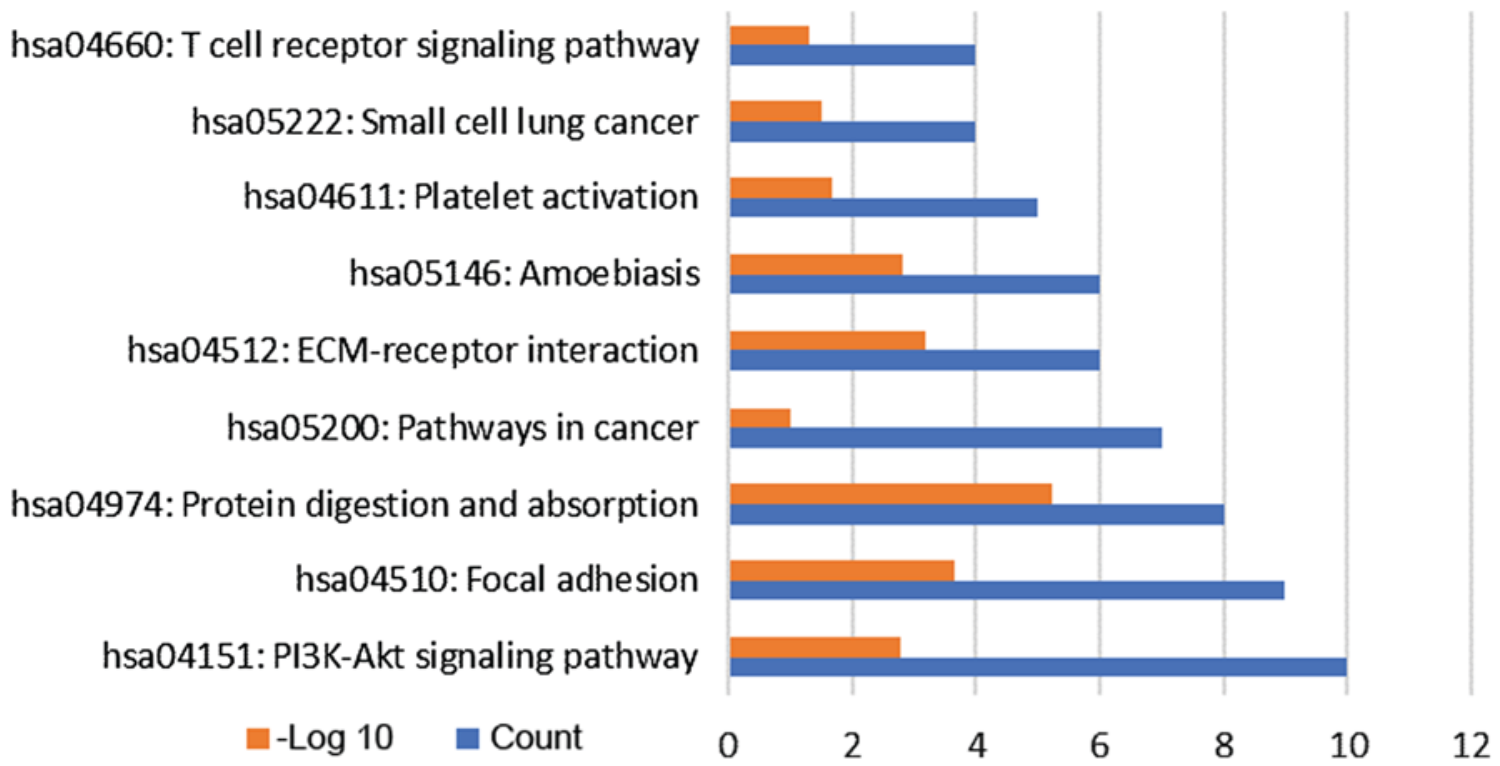

Figure 4. Kyoto Encyclopedia of Genes and Genomes signaling pathway enrichment of target genes. Red indicates the-log P of the enriched pathway and blue indicates the counts of genes enriched in each pathway. PI3K, phosphoinositide-3 kinase; ECM, extracellular matrix; hsa, Homo sapiens.

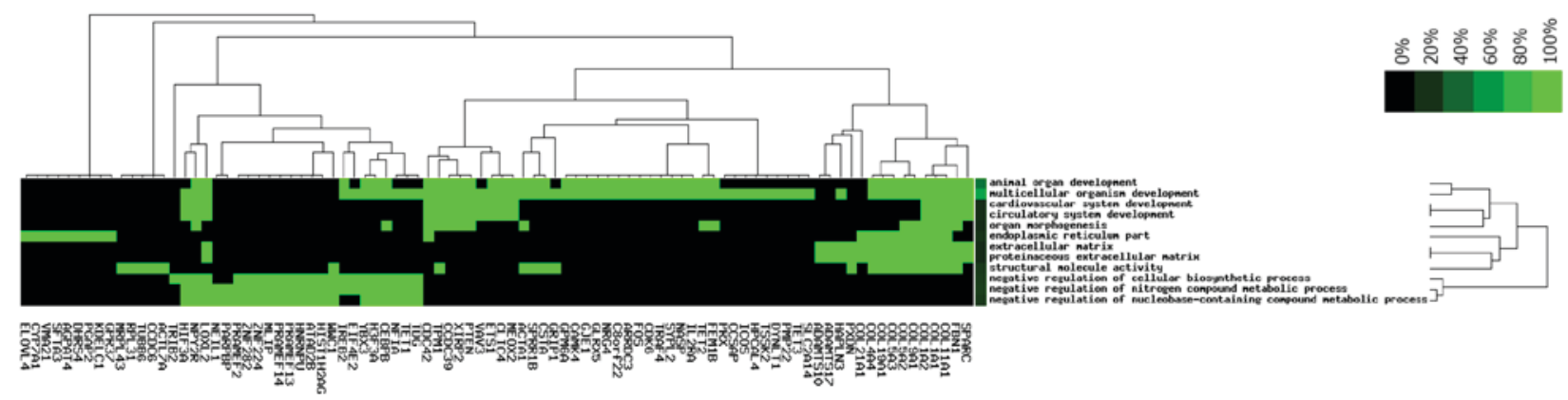

Figure 5. Gene Ontology analysis of the target genes. The color scale illustrates the corresponding gene-term associations. Green indicates the presence of a corresponding gene-term association and black indicates the absence of a corresponding gene-term association in the database.

analysis has been widely used to investigate DEMs, dysregulated genes and pathways associated with LTBI $(21,30,31)$. In previous decades, a large amount of research investigating this matter has emerged (18,32-34); however, identifying novel miRs can be used to investigate further the underlying mechanism of LTBI. Thus, in the present study, expression data obtained from patients with and without LTBI were subjected to a bioinformatics analysis to provide more reliable data.

In the analysis of the present study, 2 datasets were included. A total of 144 DEMs were identified from these 2 datasets. By exploring the overlapping miRs in the two datasets, hsa-miR-29a and hsa-miR-15b were identified as being decreased, while hsa-miR-576-5p, hsa-miR-500 and hsa-miR-155 were identified to be upregulated. Of note, miR-29a was previously reported to be significantly decreased in patients with LTBI $(35,36)$. miRs encoded by the miR-15b/16-2 cluster may act as tumor suppressors. Aberrant regulation of miR-15b in human cancers reportedly has an important role in cancer development, contributing to reduced proliferation, cell death, angiogenesis and metabolic reprogramming and metastasis resistance, as well as tumor-associated inflammation and genomic instability (37). miR-576 is directly targeted by hepatitis B virus-encoded X protein (38). miR-155 and miR-500 were reported as potential markers of aflatoxin exposure (39), which suggests that miRs may be used as potential biomarkers for the diagnosis of LTBI. However, the possible association of hsa-miR-15b, hsa-miR-576-5p, hsa-miR-500 and hsa-miR-155 with LTBI identified in the present analysis remains to be verified.

To gain a broad understanding of the effects of miRs in LTBI, the target genes of the 5 abovementioned DEMs were predicted. By merging the miR regulatory network and protein-protein interaction network, a degree of crosstalk among these miRs via cancer-associated genes, including FOS, ACTA1, CDC42 and PTEN, was identified (40-43). In the current study, pathways associated with small cell lung cancer also appeared in the KEGG pathway analysis, which suggests that LTBI may participate in the development of small cell lung cancer. By exploring the topological structure of the interaction network, it was revealed that hsa-miR-500a-3p and hsa-miR-29a-3p, as well as the 4 genes CDC42, ACTA1, PTEN and FOS, have essential roles in this regulatory network. These miRs and genes may be core factors in the diagnosis of LTBI.

To determine the molecular mechanisms underlying the LTBI process, GO term and KEGG pathway analysis 
Table III. Target gene prediction of miRs by TargetScan.

\begin{tabular}{cc}
\hline Cumulative weighted \\
context++ score
\end{tabular}

hsa-miR-29a-3p

$$
\text { COL1A1 }
$$

TET3

TET1

TET2

ATAD2B

HRK

TRIB2

SUV420H2

COL1A2

NFIA

EIF4E2

TIMM8B

TDG

IFI30

COL11A1

C7orf73

LYSMD1

COL5A3

SMIM17

IREB2

YPEL2

NASP

SPARC

ZKSCAN4

SLC30A3

PMP22

SYPL2

SMTNL2

DTWD2

TMEM236

TMEM236

ZBTB34

GPR37

ENHO

ICOS

HIF3A

ADAMTS17

SFTA3

REV3L

COL5A2

COL4A4

COL21A1

COL9A1

CCSAP

ARRDC3

FBN1

CDC42

PGAP2

TSPAN14

SESTD1
Table III. Continued.

\begin{tabular}{|c|c|}
\hline miR/gene symbol & $\begin{array}{c}\text { Cumulative weighted } \\
\text { context++ score }\end{array}$ \\
\hline LOXL2 & -0.57 \\
\hline TMEM183A & -0.56 \\
\hline TPM1 & -0.56 \\
\hline HAPLN3 & -0.56 \\
\hline MLIP & -0.55 \\
\hline GSTA4 & -0.55 \\
\hline MEX3B & -0.55 \\
\hline ZNF282 & -0.55 \\
\hline COL19A1 & -0.55 \\
\hline YBX3 & -0.55 \\
\hline ISG20L2 & -0.55 \\
\hline TRAF4 & -0.54 \\
\hline SH3BP5L & -0.54 \\
\hline PTEN & -0.53 \\
\hline RNF39 & -0.53 \\
\hline KDELC1 & -0.53 \\
\hline KIAA1024 & -0.53 \\
\hline ELOVL4 & -0.53 \\
\hline RAET1L & -0.53 \\
\hline PXDN & -0.53 \\
\hline GRIP1 & -0.52 \\
\hline FEM1B & -0.52 \\
\hline WDFY1 & -0.52 \\
\hline TBC1D7 & -0.52 \\
\hline AGPAT4 & -0.52 \\
\hline KCTD5 & -0.52 \\
\hline ADAMTS10 & -0.52 \\
\hline FAM167A & -0.51 \\
\hline \multicolumn{2}{|l|}{ hsa-miR-15b-3p } \\
\hline PGR & -1 \\
\hline PRB 1 & -0.89 \\
\hline OR2J3 & -0.86 \\
\hline PRB2 & -0.77 \\
\hline PRB3 & -0.74 \\
\hline NPY2R & -0.71 \\
\hline C1QL3 & -0.68 \\
\hline CYP7A1 & -0.66 \\
\hline DYNLT1 & -0.65 \\
\hline GLRX5 & -0.64 \\
\hline SPRR1B & -0.62 \\
\hline C5orf30 & -0.61 \\
\hline TUBB6 & -0.59 \\
\hline NSMCE4A & -0.58 \\
\hline COQ4 & -0.58 \\
\hline RP11-422N16.3 & -0.56 \\
\hline CSTA & -0.56 \\
\hline TAS2R1 & -0.54 \\
\hline COA5 & -0.54 \\
\hline OR4C13 & -0.52 \\
\hline HDDC2 & -0.52 \\
\hline C12orf60 & -0.51 \\
\hline
\end{tabular}


Table III. Continued.

\begin{tabular}{|c|c|}
\hline miR/gene symbol & $\begin{array}{c}\text { Cumulative weighted } \\
\text { context++ score }\end{array}$ \\
\hline C6ORF50 & -0.51 \\
\hline PARPBP & -0.51 \\
\hline COMMD10 & -0.51 \\
\hline \multicolumn{2}{|l|}{ hsa-miR-576-5p } \\
\hline HNRNPU & -1 \\
\hline HIST1H2AG & -0.80 \\
\hline C4orf22 & -0.66 \\
\hline C8orf22 & -0.63 \\
\hline CCDC148 & -0.61 \\
\hline CCDC39 & -0.60 \\
\hline AC069547.1 & -0.59 \\
\hline LIPT1 & -0.59 \\
\hline MS4A12 & -0.55 \\
\hline RPL31 & -0.54 \\
\hline ENY2 & -0.54 \\
\hline GJE1 & -0.53 \\
\hline RBAK-RBAKDN & -0.51 \\
\hline \multicolumn{2}{|l|}{ hsa-miR-500a-3p } \\
\hline PRX & -0.95 \\
\hline RP11-455G16.1 & -0.84 \\
\hline PRAMEF2 & -0.77 \\
\hline TSSK2 & -0.77 \\
\hline SLC2A14 & -0.73 \\
\hline NKX2-3 & -0.72 \\
\hline DHRS4 & -0.70 \\
\hline C3orf55 & -0.68 \\
\hline RP11-934B9.3 & -0.67 \\
\hline DHRS4L2 & -0.66 \\
\hline ВX088651.1 & -0.66 \\
\hline NRG4 & -0.65 \\
\hline NEIL1 & -0.65 \\
\hline SMIM5 & -0.64 \\
\hline TVP23B & -0.64 \\
\hline $\mathrm{RP} 2$ & -0.64 \\
\hline C17orf58 & -0.64 \\
\hline PPP4R1 & -0.63 \\
\hline KLHL34 & -0.63 \\
\hline CAMK4 & -0.63 \\
\hline CLIC4 & -0.63 \\
\hline CDK6 & -0.63 \\
\hline IL2RA & -0.62 \\
\hline BTF3L4 & -0.61 \\
\hline PRAMEF14 & -0.61 \\
\hline HPCAL4 & -0.61 \\
\hline ZNF98 & -0.61 \\
\hline SOCS2 & -0.60 \\
\hline PRAMEF13 & -0.60 \\
\hline XIRP2 & -0.60 \\
\hline ZNF711 & -0.60 \\
\hline PLSCR5 & -0.59 \\
\hline SLC2A3 & -0.58 \\
\hline
\end{tabular}

Table III. Continued.

\begin{tabular}{|c|c|}
\hline miR/gene symbol & $\begin{array}{c}\text { Cumulative weighted } \\
\text { context++ score }\end{array}$ \\
\hline AC006372.1 & -0.58 \\
\hline AP3B2 & -0.56 \\
\hline MRPL43 & -0.56 \\
\hline NGDN & -0.56 \\
\hline MRFAP1L1 & -0.56 \\
\hline CCDC6 & -0.55 \\
\hline CDH19 & -0.55 \\
\hline PMAIP1 & -0.55 \\
\hline ZNF224 & -0.54 \\
\hline ANKRD10 & -0.54 \\
\hline AKR1CL1 & -0.54 \\
\hline ADI1 & -0.53 \\
\hline ING1 & -0.53 \\
\hline NIPAL3 & -0.52 \\
\hline KCNJ1 & -0.52 \\
\hline GPM6A & -0.52 \\
\hline NXT2 & -0.52 \\
\hline ANO2 & -0.52 \\
\hline RP11-366L20.2 & -0.51 \\
\hline MEOX2 & -0.51 \\
\hline CCNI2 & -0.51 \\
\hline \multicolumn{2}{|l|}{ hsa-miR-155-5p } \\
\hline ZNF385D & -0.80 \\
\hline TMPRSS11BNL & -0.68 \\
\hline VAV3 & -0.64 \\
\hline ACTA1 & -0.59 \\
\hline ARID2 & -0.58 \\
\hline H3F3A & -0.58 \\
\hline ETS1 & -0.57 \\
\hline ZNF652 & -0.57 \\
\hline VMA21 & -0.54 \\
\hline ACTL7A & -0.53 \\
\hline TM6SF1 & -0.53 \\
\hline CEBPB & -0.52 \\
\hline FOS & -0.52 \\
\hline WWC1 & -0.52 \\
\hline TAOK1 & -0.51 \\
\hline
\end{tabular}

miR, microRNA; hsa, Homo sapiens.

were included in the present study to perform a comprehensive analysis of the roles of the key miRs and genes. In the KEGG pathway enrichment analysis, 'PI3K/AKT signaling pathway' appeared to be the most enriched pathway with the largest number of enriched genes. The PI3K/AKT signaling pathway, including its downstream pathway, mammalian target of rapamycin (mTOR), is well known to be involved in a variety of BPs, and it has been reported that hyperactivation of mTOR has a pathogenetic role in human immunodeficiency virus infection (44-46). Due to the important role of the AKT 
pathway in cellular metabolism, growth and division, apoptosis suppression and angiogenesis, the regulation of the AKT signaling may represent a valuable therapeutic strategy. For this reason, AKT inhibitors have become the hotspots of research in a number of clinical diseases (47), and dual PI3K/mTOR inhibitors, including PF-04691502 and NVP-BEZ235, may have important therapeutic applications in cancer $(48,49)$. Anti-retroviral protease inhibitors have been re-purposed as inhibitors of the PI3K/AKT/mTOR pathway, and such drugs that are also available as a generic and cheap formulation may be immediately available for testing in patients with LTBI (50). Studies have also indicated on upregulated activation of the $\mathrm{PI} 3 \mathrm{~K} / \mathrm{AKT} / \mathrm{mTOR}$ pathway in autoimmune diseases, including multiple sclerosis and liver fibrosis $(51,52)$. It has been demonstrated that the PI3K/AKT signaling pathway is inhibited in patients with active TB $(53,54)$. However, there is no direct evidence indicating PI3K/AKT signaling pathway alterations in patients with LTBI. 'Protein digestion and absorption' was the most significant pathway. These signaling pathways constitute important potential mechanisms underlying the processes of LTBI and merit further validation.

In conclusion, the present analysis identified hsa-miR-500a-3p and hsa-miR-29a-3p, as well as 4 target genes CDC42, ACTA1, PTEN and FOS, which may be used as potential biomarkers of LTBI. The PI3K/AKT signaling pathway is the key genetic event implicated in LTBI, and an in-depth investigation of the efficiency of PI3K/AKT signaling inhibitors in the prevention of LTBI is warranted. The novel key factors and molecular pathways provided in the present study may contribute to the current understanding of LTBI and may facilitate the development of a molecular diagnostic platform for its detection.

\section{Acknowledgements}

Not applicable.

\section{Funding}

The present study was supported by the National Natural Science Foundation of China (grant no. 81660330).

\section{Availability of data and materials}

All datasets used and/or analyzed during the present study are available from the corresponding author on reasonable request.

\section{Authors' contributions}

YL, HD, XiaW, PY, LH, YW, ZZ and WZ analyzed the data. YL, XinW, HD and LZ designed the study and prepared the manuscript.

\section{Ethical approval and consent to participate}

Not applicable.

\section{Patient consent for publication}

Not applicable.

\section{Competing interests}

The authors declare that they have no competing interest.

\section{References}

1. WHO:Globaltuberculosisreport2017.http://wwwwhoint/tb/publications/global_report/en/, 2017.

2. WHO: Latent tuberculosis infection Updated and consolidated guidelines for programmatic management. http://appswhoint/iris/bitstream/10665/260233/1/978924155023 9-engpdf?ua=1, 2018.

3. Palomino JC, Martin A, Von Groll A and Portaels F: Rapid culture-based methods for drug-resistance detection in Mycobacterium tuberculosis. J Microbiol Methods 75: 161-166, 2008.

4. Tang P and Johnston J: Treatment of latent tuberculosis infection. Curr Treat Options Infect Dis 9: 371-379, 2017.

5. Alvarez-León EE, Espinosa-Vega E, Santana-Rodriguez E, Molina-Cabrillana JM, Pérez-Arellano JL, Caminero JA and Serrano-Aguilar P: Screening for tuberculosis infection in spanish healthcare workers: Comparison of the QuantiFERON-TB gold in-tube test with the tuberculin skin test. Infect Control Hosp Epidemiol 30: 876-883, 2009.

6. Menzies D, Pai M and Comstock G: Meta-analysis: New tests for the diagnosis of latent tuberculosis infection: Areas of uncertainty and recommendations for research. Ann Intern Med 146: 340-354, 2007.

7. Jiang W, Shao L, Zhang Y, Zhang S, Meng C, Xu Y, Huang L, Wang Y, Wang Y, Weng X and Zhang W: High-sensitive and rapid detection of Mycobacterium tuberculosis infection by IFN-gamma release assay among HIV-infected individuals in BCG-vaccinated area. BMC Immunol 10: 31, 2009.

8. Matulis G, Juni P, Villiger PM and Gadola SD: Detection of latent tuberculosis in immunosuppressed patients with autoimmune diseases: Performance of a Mycobacterium tuberculosis antigen-specific interferon gamma assay. Ann Rheum Dis 67: 84-90, 2008

9. WHO: Guidelines on the management of latent tuberculosis infection. http://wwwwhoint/tb/publications/latent-tuberculosis-infection/en/, 2014.

10. Bartel DP: MicroRNAs: Genomics, biogenesis, mechanism, and function. Cell 116: 281-297, 2004.

11. Lukiw WJ: Micro-RNA speciation in fetal, adult and Alzheimer's disease hippocampus. Neuroreport 18: 297-300, 2007.

12. Hayes J, Peruzzi PP and Lawler S: MicroRNAs in cancer: Biomarkers, functions and therapy. Trends Mol Med 20: 460-469, 2014.

13. Chen H, Lan HY, Roukos DH and Cho WC: Application of microRNAs in diabetes mellitus. J Endocrinol 222: R1-R10, 2014.

14. Kumarswamy $\mathrm{R}$ and Thum T: Non-coding RNAs in cardiac remodeling and heart failure. Circ Res 113: 676-689, 2013.

15. Jin BX, Zhang YH, Jin WJ, Sun XY, Qiao GF, Wei YY, Sun LB, Zhang WH and Li N: MicroRNA panels as disease biomarkers distinguishing hepatitis B virus infection caused hepatitis and liver cirrhosis. Sci Rep 5: 15026, 2015.

16. Staedel C and Darfeuille F: MicroRNAs and bacterial infection. Cellular microbiology 15: 1496-1507, 2013.

17. Wu LS, Lee SW, Huang KY, Lee TY, Hsu PW and Weng JT: Systematic expression profiling analysis identifies specific microRNA-gene interactions that may differentiate between active and latent tuberculosis infection. Biomed Res Int 2014: 895179, 2014.

18. Meng QL, Liu F, Yang XY, Liu XM, Zhang X, Zhang C1 and Zhang ZD: Identification of latent tuberculosis infection-related microRNAs in human U937 macrophages expressing Mycobacterium tuberculosis Hsp16.3. BMC Microbiol 14: 37, 2014.

19. Rai G, Rai R, Saeidian AH and Rai M: Microarray to deep sequencing: Transcriptome and miRNA profiling to elucidate molecular pathways in systemic lupus erythematosus. Immunol Res 64: 14-24, 2016.

20. Zheng Y, Qing T, Song Y, Zhu J, Yu Y, Shi W, Pusztai L and Shi L: Standardization efforts enabling next-generation sequencing and microarray based biomarkers for precision medicine. Biomark Med 9: 1265-1272, 2015.

21. Zheng L, Leung E, Lee N, Lui G, To KF, Chan RC and Ip M: Differential microRNA expression in human macrophages with Mycobacterium tuberculosis infection of Beijing/W and NON-Beijing/W strain types. PLoS One 10: e0126018, 2015. 
22. Gentleman RC, Carey VJ, Bates DM, Bolstad B, Dettling M, Dudoit S, Ellis B, Gautier L, Ge Y, Gentry J, et al: Bioconductor: Open software development for computational biology and bioinformatics. Genome Biol 5: R80, 2004.

23. Mizrachi E, Verbeke L, Christie N, Fierro AC, Mansfield SD, Davis MF, Gjersing E, Tuskan GA, Van Montagu M, Van de Peer Y, et al: Network-based integration of systems genetics data reveals pathways associated with lignocellulosic biomass accumulation and processing. Proc Natl Acad Sci USA 114: 1195-1200, 2017.

24. Agarwal V, Bell GW, Nam JW and Bartel DP: Predicting effective microRNA target sites in mammalian mRNAs. Elife 4: 2015

25. Szklarczyk D, Franceschini A, Wyder S, Forslund K, Heller D, Huerta-Cepas J, Simonovic M, Roth A, Santos A, Tsafou KP, et al: STRING v10: Protein-protein interaction networks, integrated over the tree of life. Nucleic Acids Res 43 (Database Issue) D447-D452, 2015.

26. Muetze T, Goenawan IH, Wiencko HL, Bernal-Llinares M, Bryan K and Lynn DJ: Contextual hub analysis tool (CHAT): A Cytoscape app for identifying contextually relevant hubs in biological networks. F1000 Res 5: 1745, 2016.

27. Su G, Morris JH, Demchak B and Bader GD: Biological network exploration with Cytoscape 3. Curr Protoc Bioinformatics 47: 8.13.1-24, 2014.

28. Du J, Yuan Z, Ma Z, Song J, Xie X and Chen Y: KEGG-PATH: Kyoto encyclopedia of genes and genomes-based pathway analysis using a path analysis model. Mol Biosyst 10: 2441-2447, 2014.

29. Wang JH, Zhao LF, Lin P, Su XR, Chen SJ, Huang LQ, Wang HF, Zhang H, Hu ZF, Yao KT and Huang ZX: GenCLiP 2.0: A web server for functional clustering of genes and construction of molecular networks based on free terms. Bioinformatics 30 2534-2536, 2014.

30. Xin H, Yang Y, Liu J, Li X, Li M, Feng B, Li Z, Zhang H, Li H, Shen F, et al: Association between tuberculosis and circulating microRNA hsa-let-7b and hsa-miR-30b: A pilot study in a Chinese population. Tuberculosis (Edinb) 99: 63-69, 2016.

31. Fu Y, Yi Z, Li J and Li R: Deregulated microRNAs in CD4+ $\mathrm{T}$ cells from individuals with latent tuberculosis versus active tuberculosis. J Cell Mol Med 18: 503-513, 2014.

32. Wang C, Yang S, Sun G, Tang X, Lu S, Neyrolles O and Gao Q: Comparative miRNA expression profiles in individuals with latent and active tuberculosis. PLoS One 6: e25832, 2011.

33. Ndzi EN, Nkenfou CN, Mekue LM, Zentilin L, Tamgue O, Pefura EWY, Kuiaté JR, Giacca M and Ndjolo A: MicroRNA hsa-miR-29a-3p is a plasma biomarker for the differential diagnosis and monitoring of tuberculosis. Tuberculosis (Edinb) 114 69-76, 2019.

34. Sabir N, Hussain T, Shah SZA, Peramo A, Zhao D and Zhou X: miRNAs in tuberculosis: New avenues for diagnosis and host-directed therapy. Front Microbiol 9: 602, 2018.

35. Kleinsteuber K, Heesch K, Schattling S, Kohns M, Sander-Jülch C, Walzl G, Hesseling A, Mayatepek E, Fleischer B, Marx FM and Jacobsen M: Decreased expression of miR-21, miR-26a, miR-29a, and miR-142-3p in CD4 ${ }^{+} \mathrm{T}$ cells and peripheral blood from tuberculosis patients. PLoS One 8: e61609, 2013.

36. Pan D, Pan M and Xu YM: Mir-29a expressions in peripheral blood mononuclear cell and cerebrospinal fluid: Diagnostic value in patients with pediatric tuberculous meningitis. Brain Res Bull 130: 231-235, 2017.

37. Zhao C, Wang G, Zhu Y, Li X, Yan F, Zhang C, Huang X and Zhang Y: Aberrant regulation of miR-15b in human malignant tumors and its effects on the hallmarks of cancer. Tumour Biol 37: 177-183, 2016.

38. Guerrieri F, Belloni L, D'Andrea D, Pediconi N, Le Pera L, Testoni B, Scisciani C, Floriot O, Zoulim F, Tramontano A and Levrero M: Genome-wide identification of direct HBx genomic targets. BMC Genomics 18: 184, 2017.
39. Valencia-Quintana R, Sánchez-Alarcón J, Tenorio-Arvide MG, Deng Y, Montiel-González JM, Gómez-Arroyo S, Villalobos-Pietrini R, Cortés-Eslava J, Flores-Márquez AR and Arenas-Huertero F: The microRNAs as potential biomarkers for predicting the onset of aflatoxin exposure in human beings: A review. Front Microbiol 5: 102, 2014.

40. Aguilar BJ, Zhou H and Lu Q: Cdc42 signaling pathway inhibition as a therapeutic target in ras- related cancers. Curr Med Chem 24: 3485-3507, 2017.

41. Chang H, Sung JH, Moon SU, Kim HS, Kim JW and Lee JS: EGF Induced RET inhibitor resistance in CCDC6-RET lung cancer cells. Yonsei Med J 58: 9-18, 2017.

42. Kato F,FiorentinoFP,Alibés A,Perucho M,Sánchez-Céspedes M, Kohno T and Yokota J: MYCL is a target of a BET bromodomain inhibitor, JQ1, on growth suppression efficacy in small cell lung cancer cells. Oncotarget 7: 77378-77388, 2016.

43. Cui M, Augert A, Rongione M, Conkrite K, Parazzoli S, Nikitin AY, Ingolia N and MacPherson D: PTEN is a potent suppressor of small cell lung cancer. Mol Cancer Res 12: 654-659, 2014

44. Nicoletti F, Fagone P, Meroni P, McCubrey J and Bendtzen K: mTOR as a multifunctional therapeutic target in HIV infection. Drug Discov Today 16: 715-721, 2011.

45. Nicoletti F, Lapenta C, Donati S, Spada M, Ranazzi A, Cacopardo B, Mangano K, Belardelli F, Perno C and Aquaro S: Inhibition of human immunodeficiency virus (HIV-1) infection in human peripheral blood leucocytes-SCID reconstituted mice by rapamycin. Clin Exp Immunol 155: 28-34, 2009.

46. Donia M, McCubrey JA, Bendtzen K and Nicoletti F: Potential use of rapamycin in HIV infection. Br J Clin Pharmacol 70: 784-793, 2010

47. Nitulescu GM, Van De Venter M, Nitulescu G, Ungurianu A Juzenas P, Peng Q, Olaru OT, Grădinaru D, Tsatsakis A and Tsoukalas D, et al: The Akt pathway in oncology therapy and beyond (Review). Int J Oncol 53: 2319-2331, 2018.

48. Chen D, Mao C, Zhou Y, Su Y, Liu S and Qi WQ: PF-04691502, a dual PI3K/mTOR inhibitor has potent pre-clinical activity by inducing apoptosis and G1 cell cycle arrest in aggressive B-cell non-Hodgkin lymphomas. Int J Oncol 48: 253-260, 2016.

49. Moon du G, Lee SE, Oh MM, Lee SC, Jeong SJ, Hong SK, Yoon CY, Byun SS, Park HS and Cheon J: NVP-BEZ235, a dual PI3K/mTOR inhibitor synergistically potentiates the antitumor effects of cisplatin in bladder cancer cells. Int J Oncol 45: 1027-1035, 2014

50. Maksimovic-Ivanic D, Fagone P, McCubrey J, Bendtzen K, Mijatovic S and Nicoletti F: HIV-protease inhibitors for the treatment of cancer: Repositioning HIV protease inhibitors while developing more potent NO-hybridized derivatives? Int J Cancer 140: 1713-1726, 2017.

51. Mammana S, Bramanti P, Mazzon E, Cavalli E, Basile MS, Fagone P, Petralia MC, McCubrey JA, Nicoletti F and Mangano K: Preclinical evaluation of the PI3K/Akt/mTOR pathway in animal models of multiple sclerosis. Oncotarget 9: 8263-8277, 2018.

52. Fagone P, Mangano K, Pesce A, Portale TR, Puleo S and Nicoletti F: Emerging therapeutic targets for the treatment of hepatic fibrosis. Drug Discov Today 21: 369-375, 2016.

53. Wang FY, Wang XM, Wang C, Wang XF, Zhang YQ, Wu JD, Wu F, Zhang WJ and Zhang L: Suppression of Mcl-1 induces apoptosis in mouse peritoneal macrophages infected with Mycobacterium tuberculosis. Microbiol Immunol 60: 215-227, 2016.

54. Kim KH, Yang CS, Shin AR, Jeon SR, Park JK, Kim HJ and Jo EK: Mycobacterial heparin-binding hemagglutinin antigen activates inflammatory responses through PI3-K/Akt, NF- $\mathrm{KB}$, and MAPK pathways. Immune Netw 11: 123-133, 2011.

This work is licensed under a Creative Commons Attribution-NonCommercial-NoDerivatives 4.0 International (CC BY-NC-ND 4.0) License. 